\title{
Studying the relationship between a woman's reproductive lifespan and age at menarche using a Bayesian multivariate structured additive distributional regression model
}

\author{
Elisa Duarte ${ }^{*, 1}$, Bruno de Sousa $^{2}$, Carmen Cadarso-Suárez ${ }^{1}$, Nadja Klein ${ }^{3}$, Thomas Kneib \\ ${ }^{3}$, and Vítor Rodrigues ${ }^{4}$ \\ ${ }^{1}$ Unit of Biostatistics, Department of Statistics, Mathematical Analysis, and Optimization, School of \\ Medicine University of Santiago de Compostela, C/ San Francisco s/n, 15782 Santiago de Compostela, \\ Spain \\ ${ }^{2}$ Faculty of Psychology and Education Sciences, University of Coimbra, CINEICC, Rua do Colégio Novo, \\ Apartado 6153, 3001-802 Coimbra, Portugal \\ ${ }^{3}$ Institute of Statistics and Econometrics, Dept. of Economics, Georg-August-Universität Göttingen, \\ Humboldtallee 3, 37073 Göttingen, Germany \\ ${ }^{4}$ Faculty of Medicine, University of Coimbra, Rua Larga, 3004-504 Coimbra, Portugal
}

Received zzz, revised zzz, accepted zzz

\begin{abstract}
Studies addressing breast cancer risk factors have been looking at trends relative to age at menarche and menopause. These studies point to a downward trend of age at menarche and an upward trend for age at menopause, meaning an increase of a woman's reproductive lifespan cycle. In addition to studying the effect of the year of birth on the expectation of age at menarche and a woman's reproductive lifespan, it is important to understand how a woman's cohort affects the correlation between these two variables. Since the behavior of age at menarche and menopause may vary with the geographic location of a woman's residence, the spatial effect of the municipality where a woman resides needs to be considered. Thus, a Bayesian multivariate structured additive distributional regression model is proposed in order to analyze how a woman's municipality and year of birth affects a woman's age of menarche, her lifespan cycle, and the correlation of the two. The data consists of 212,517 post menopause women, born between 1920 and 1965, who attended the breast cancer screening program in the central region of Portugal.
\end{abstract}

Key words: Bayesian multivariate structured additive distributional regression model; Menarche age; Reproductive lifespan; Spatial effects

\section{Introduction}

Several studies (Hsieh et al., 1990; Kelsey et al., 1993; McPherson et al., 2000; Lambe, 2010) over the years have pointed to early menarche and late menopause as important breast cancer risk factors. This has triggered studies aiming to analyze possible time trends regarding a woman's age at menarche and menopause (Talma et al., 2013; Berman et al., 2012; Rigon et al., 2010; Cabanes et al., 2009; Nichols, 2006; Padez and Rocha, 2003). In general, these studies describe a significant decrease in age of menarche over time, without clear evidence regarding an increase of a woman's age at menopause. This particular problem was addressed by the authors in an earlier study (Duarte et al., 2014), where they investigated how age of menarche and menopause relates to a woman's year of birth, together with other breast cancer risk factors such as whether a woman has been pregnant at least once in her life, and whether she breastfed, among others. Also considered were the possible spatial correlations that can arise from a woman's place

${ }^{*}$ Corresponding author: e-mail: duarte.elisa@gmail.com, Phone: +351-917-240-783 
of residence. The findings showed that not only early menarche is observed for younger women, i.e., the ones with the most recent year of birth, but also a decreasing time trend of the age of menopause seemed to prevail along the birth cohorts under study.

Rather than studying the evolution of the age of menarche and menopause separately, it is worth taking up their relationship jointly. Since these two moments mark the beginning and the ending of a woman's lifetime exposure to endogenous hormones, a variable that can be taken into consideration is a woman's reproductive lifespan cycle, determined by the difference between a woman's age at menopause and menarche. A reference to this variable is made by Nichols (2006), where the results pointed to an upward trend in the number of a woman's reproductive years, but in which the decreasing trend of both age at menarche and menopause found in Duarte et al. (Duarte et al., 2014) does not seem to corroborate Nichols' findings. In addition, notice that identical reproductive lifespans of a woman does not mean that their cycle occurred at the same point in time, thus indicating that it is very important to consider the cohort to which a woman belongs. Therefore, in this work we propose a model to study the effect of the year of birth on a bivariate response, consisting of a woman's age at menarche and her reproductive lifespan cycle. Age at menarche was chosen instead of age at menopause because, although both events depend on a woman's memory, menarche is a singular life-changing event in a woman's adolescence, more so than menopause, which can be considered a process extended over time, thus making it difficult to pinpoint a specific date for its occurrence. Another reason for this choice of variables is determined by the results of the study carried out by the Collaborative Group on Hormonal Factors on Breast Cancer (2012) which confirmed that early menarche and late menopause increase breast cancer risk, but where early menarche constitutes a greater breast cancer risk than late menopause.

Therefore, it should be of the utmost importance to explore how individual characteristics such as age at menarche and a woman's reproductive lifespan can be a reflection of influence from any environmental factors. This matter is addressed in the present work by including the spatial effect of a woman's place of residence as a predictor covariate in our multivariate model.

To sum up, the aim of this study is to explore the effect of the birth cohort and of a woman's social and natural environment of where she resides on the correlation of the two individual variables: age at menarche age and a woman's reproductive lifespan cycle. Thus, the problem requires regression models to be formulated in a multivariate way, where correlated response variables can be considered simultaneously using a structured additive predictor. The regression models performed in this study are based on a Bayesian structured additive distributional regression methodology for multivariate response developed by Klein et al. (2015b). This unified Bayesian approach for a multivariate structured additive distributional regression is an extension of the work developed by the same authors for univariate responses (Klein $e t$ al., 2015a), where the main idea is to model other parameters of the response distribution rather than the usual mean. Section 3 of this paper will give a brief description of this methodology. The rest of the paper is structured as follows: Section 2 presents the data set used in this study, Section 4 provides the results of the proposed analysis, followed by the discussion in Section 5 .

\section{The breast cancer screening data}

The data used in this study was provided by the Central Regional Nucleus of the Portuguese Cancer League (LPCC-NRC), sponsored by the Breast Cancer Screening Program (BCSP) in 78 municipalities located in central Portugal's. The database consists of women who were registered for the BCSP in central Portugal between 1990 and 2010. The screening program is implemented gradually over central Portugal thus, in a given year, there are municipalities in different rounds of the screening. In every follow-up round the information registered in previous rounds is revised and confirmed. Even if a woman has been diagnosed with a tumor, which means she is entrusted to the National Health Services and leaves the screening program, her last round information remains in the database and is part of this study. For this 
reason, the most recent data collected from the last visit that a woman made to the screening program is the one being studied.

In Portugal, participation in the screening program takes place via invitation and is not mandatory, implying some absenteeism that can lead to bias in the results due to the different levels of the municipalities' attendance rates. The complexity of the data collected throughout these last 20 years is pictured in Figure 1, which shows the evolution of the attendance rates for municipalities, separated by 4 different levels of rate of attendance when they first entered the screening program, and where colors represent the year in which a municipality entered the program. Looking at this figure, there is a clear improvement of the attendance rates in the central region of Portugal throughout the years. The majority of the municipalities started with attendance rates higher that $30 \%$, with rates reaching more than $70 \%$ over $57 \%$ of the municipalities.

[Figure 1 here]

The data set is an extension of the data used in Duarte et al. (2014) with 278,282 registries. This new data set not only includes the previous information, but also the information concerning the screening rounds of three additional years as compared to the first one. Therefore, women considered in this study have a screening age between 45 and 69 , with $76 \%(212,517)$ of them reaching menopause.

The variables used in this study are: age of menarche, a woman's reproductive lifespan cycle (calculated by subtracting the age of menarche from the age of menopause), year of birth, and the code of the municipality where a woman resides. A summary description of these variables can be found in Table 1 .

[Table 1 here]

As mentioned above, the reproductive lifespan cycle is the difference between the age at menopause and the age at menarche. Although, the database consists not only of post-menopausal women, but also of women that have not yet reached the menopause. To take in account the latter cases, i.e. pre-menopausal women, we would have to develop in future research an extension of Bayesian multivariate structured additive regression models that will take into account censored responses. Thus, only post-menopausal women were considered in this study. Consequently, they are representative of the group of women who are older and have remained in the screening program for some time, thus excluding younger women that have not yet entered menopause as well as those who dropped out of the screening program before entering menopause. Table 2 shows the distribution of the women grouped into three cohorts. The first cohort contains women born before 1931, the second covers women born between 1931 and 1955, and the third cohort includes those women born after 1955. Looking at the frequency table, it is clear that the majority of the women (86\%) are in the second cohort, $1931-1955$. In the following section, by proposing a model that includes varying coefficients, the main cohort of women $(1931-1955)$ is separated from remaining cohorts, thus minimizing the selection bias that is usually present in the screening program.

[Table 2 here]

\section{Model formulation}

As previously mentioned, the aim of this work is to understand to what extent a woman's year of birth and the region where she resides are associated with her age of menarche, her reproductive lifespan cycle, and the possible correlation between the two. With this in mind, the model performed has a bivariate response, the pair age at menarche and a woman's reproductive lifespan. From this point onward, in the interest of simplifying the notation, the variable reproductive lifespan cycle shall be referred to as "window". Thus, 
the response $\mathbf{y}=(M, W)^{\prime}$, where $M$ corresponds to menarche and $W$ to window, has a bivariate normal distribution $\mathbf{y} \sim N(\boldsymbol{\mu}, \boldsymbol{\Sigma})$, with density:

$$
p(M, W)=\frac{1}{\sqrt{(2 \pi)^{2} \operatorname{det}(\boldsymbol{\Sigma})}} \exp \left\{-\frac{1}{2}(\mathbf{y}-\boldsymbol{\mu})^{\prime} \boldsymbol{\Sigma}^{-1}(\mathbf{y}-\boldsymbol{\mu})\right\}
$$

where $\boldsymbol{\mu}=(E(M), E(W))^{\prime}$ is the expectation vector and the covariance matrix is given by

$$
\sum=\left(\begin{array}{cc}
\sigma_{M}^{2} & \rho \sigma_{M} \sigma_{W} \\
\rho \sigma_{M} \sigma_{W} & \sigma_{W}^{2}
\end{array}\right)
$$

with $\sigma_{M}^{2}=\operatorname{var}(M), \sigma_{W}^{2}=\operatorname{var}(W)$, and $\rho=\operatorname{corr}(M, W)$.

Considering $K$ the number of parameters of the response distribution, the motivation from the Bayesian multivariate distributional regression proposed by Klein et al. (2015b) is to construct a semiparametric structured additive predictor $\eta_{i}^{\vartheta_{k}}$ with covariates $\boldsymbol{\nu}_{i}$ linked to each response distribution parameter $\vartheta_{i k}$, with $k=1, \ldots, K$, by a monotone, twice differentiable, response function $h_{k}$. Thus, $\vartheta_{i k}=h_{k}\left(\eta_{i}^{\vartheta_{k}}\right)$ and $\eta_{i}^{\vartheta_{k}}=$ $h_{k}^{-1}\left(\vartheta_{i k}\right)$. In the present study, where the response $\mathbf{y}=(M, W)^{\prime}$, has a bivariate normal distribution, it is necessary to consider both the expectations and standard deviations of the marginal distributions, together with the correlation parameter. Each of these parameters are estimated as functions of the covariates using the following link functions:

$$
\begin{gathered}
\eta_{i}^{\mu_{M}}=\mu_{i M}, \quad \eta_{i}^{\mu_{W}}=\mu_{i W}, \\
\eta_{i}^{\sigma_{M}}=\log \left(\sigma_{i M}\right), \quad \eta_{i}^{\sigma_{W}}=\log \left(\sigma_{i W}\right), \\
\eta_{i}^{\rho}=\rho_{i} / \sqrt{ }\left(1-\rho_{i}^{2}\right) .
\end{gathered}
$$

The semiparametric predictor $\eta_{i}^{\vartheta_{k}}$, with $\vartheta_{k} \in\left\{\mu_{M}, \mu_{W}, \sigma_{M}, \sigma_{W}, \rho\right\}$ has the general form:

$$
\eta_{i}^{\vartheta_{k}}=\sum_{j=1}^{\mathbf{J}_{k}} f_{j}^{\vartheta_{k}}\left(\boldsymbol{\nu}_{i}\right),
$$

where the functions $f_{j}^{\vartheta_{k}}\left(\boldsymbol{\nu}_{i}\right)$ can represent linear functions, continuous functions, spatial effects or other type of functions as defined in Fahrmeir et al. (2004).

Since the smooth terms are defined using basis functions, the predictors in equation (1) can be written in the following generic matrix notation

$$
\boldsymbol{\eta}^{\vartheta_{k}}=\sum_{j=1}^{\mathbf{J}_{k}} \mathbf{Z}_{j}^{\vartheta_{k}} \boldsymbol{\beta}_{j}^{\vartheta_{k}},
$$

where $\boldsymbol{\eta}^{\vartheta_{k}}$ is the vector of predictors, $\left(\eta_{1}^{\vartheta_{k}}, \ldots, \eta_{n}^{\vartheta_{k}}\right), \boldsymbol{\beta}_{j}^{\vartheta_{k}}$ are vectors of the basis coefficients to be estimated, and $\mathbf{Z}_{j}^{\vartheta_{k}}$ are design matrices obtained by evaluating the basis functions at observed covariates. The coefficients $\boldsymbol{\beta}_{j}^{\vartheta_{k}}$ are estimated using Bayesian inference with MCMC simulation techniques, assuming improper Gaussian priors:

$$
p\left\{\boldsymbol{\beta}_{j}^{\vartheta_{k}} \mid\left(\tau_{j}^{\vartheta_{k}}\right)^{2}\right\} \propto\left\{\frac{1}{\left(\tau_{j}^{\vartheta_{k}}\right)^{2}}\right\}^{r k\left(\mathbf{K}_{j}^{\vartheta_{k}}\right) / 2} \exp \left\{-\frac{1}{2\left(\tau_{j}^{\vartheta_{k}}\right)^{2}}\left(\boldsymbol{\beta}_{j}^{\vartheta_{k}}\right)^{\prime} \mathbf{K}_{j}^{\vartheta_{k}} \boldsymbol{\beta}_{j}^{\vartheta_{k}}\right\} .
$$


Thus, each term $\mathbf{f}_{j}^{\vartheta_{k}}=\left(f_{j}^{\vartheta_{k}}\left(\boldsymbol{\nu}_{1}\right), \ldots, f_{j}^{\vartheta_{k}}\left(\boldsymbol{\nu}_{n}\right)\right)^{\prime}=\mathbf{Z}_{j}^{\vartheta_{k}} \boldsymbol{\beta}_{j}^{\vartheta_{k}}$ is well defined by the design matrix $\mathbf{Z}_{j}^{\vartheta_{k}}$ and the penalty matrix $\mathbf{K}_{j}^{\vartheta_{k}}$. The smoothing variance parameters $\left(\tau_{j}^{\vartheta_{k}}\right)^{2}$ are also considered as random variables to which are assigned inverse Gamma priors $\left(\tau_{j}^{\vartheta_{k}}\right)^{2} \sim I G\left(a_{j}^{\vartheta_{k}}, b_{j}^{\vartheta_{k}}\right)$, with $a_{j}^{\vartheta_{k}}=b_{j}^{\vartheta_{k}}=0.001$. To study the effect of the year of birth and possible spatial correlations on the relationship between the window and age at menarche, the following general model was defined:

$$
\eta_{i}=\alpha+f_{1}\left(\text { birth }_{i}\right)+f_{\text {spat }}\left(\text { munic }_{i}\right)
$$

where birth is the year of birth and munic is the municipality where a woman lives. The smooth function $f_{1}$ is estimated using Bayesian P-splines (Lang and Brezger, 2004; Brezger and Lang, 2006). The P-splines approach (Eilers and Marx, 1996) allows to approximate the unknown function $f_{1}$ by a polynomial spline of degree 1 with equally spaced knots over the domain of birth. The spline can be written in terms of a linear combination of B-spline basis functions. The design matrix $\mathbf{Z}$ is given by $\mathbf{B}$-spline basis functions evaluated at the observations $b_{i r t h}$. The advantage of the P-splines comparatively to the regression splines is that the number and locations of the knots are no longer critical choices, with only a reasonable number (about 20-40) required to ensure flexibility. A wiggliness penalty is introduced to prevent overfitting. In this work, a cubic spline was chosen, that is a twice continuously differentiable function giving a visually smooth function estimate. A second order random walk is used as prior for the regression coefficients with diffuse priors for initial values. The penalty matrix is of the form $\mathbf{K}=\mathbf{D}^{\prime} \mathbf{D}$, where $\mathbf{D}$ is a second order difference matrix.

The function $f_{\text {spat }}$, representing the spatial effect of the municipality, is estimated through an intrinsic Markov random field (MRF) prior (Fahrmeir and Lang, 2001; Rue and Held, 2005).

Due to the imbalance observed in the distribution of the three cohorts presented in Table 2, and trying to diminish a possible bias derived from only being considered the post-menopausal women, the spatial effect was evaluated at each cohort through the introduction of varying coefficients that enable us to model this interaction. For each cohort range defined in Table 2, a dummy variable was considered. Cohort $1(C 1)$ corresponds to women born between 1920 and 1930, cohort $2(C 2)$ to those born between 1931 and 1955, and finally cohort $3(C 3)$ for women with year of birth from 1955 onwards. With this notation the predictor equation assumes the form:

$$
\begin{aligned}
\eta_{i}= & \alpha+f_{1}\left(\text { birth }_{i}\right)+C 1_{i} * f_{\text {spat }}\left(\text { munic }_{i}\right) \\
& +C 2_{i} * f_{\text {spat }}\left(\text { munic }_{i}\right)+C 3_{i} * f_{\text {spat }}\left(\text { munic }_{i}\right) .
\end{aligned}
$$

Following the same ideas behind distributional regression, predictor (2) is applied to each response distribution parameter $\eta_{i}^{\vartheta_{k}}$, yielding the equations for each $\vartheta_{k} \in\left\{\mu_{M}, \mu_{W}, \sigma_{M}, \sigma_{W}, \rho\right\}$ :

$$
\begin{aligned}
\eta_{i}^{\vartheta_{k}}= & \alpha^{\vartheta_{k}}+f_{1}^{\vartheta_{k}}\left(\text { birth }_{i}\right)+C 1_{i} * f_{\text {spat }}^{\vartheta_{k}}\left(\text { munic }_{i}\right) \\
& +C 2_{i} * f_{\text {spat }}^{\vartheta_{k}}\left(\text { munic }_{i}\right)+C 3_{i} * f_{\text {spat }}^{\vartheta_{k}}\left(\text { munic }_{i}\right)
\end{aligned}
$$

Inference was carried out using the Markov Chain Monte Carlo (MCMC) simulation algorithm based on distribution-specific iteratively weighted least squares approximations to the full conditionals, which is implemented in BayesX software version 3.0.2 (Belitz et al., 2015) available from http://www.bayesx.org. This approach is detailed in Klein et al. (2015a) and the multivariate extension can be found in Klein et al. (2015b). Source code to reproduce the results is available as Supporting Information on the journal's web page. 


\section{Results}

The distributional multivariate models performed in the current study, is an extension of the univariate analyses presented by Duarte et al. (2014), who analyzed the effect of a woman's the year of birth on the age at menarche and menopause, separately. The present bivariate model enabled to study the effect of the year of birth and the effect of a womans place of residence in the correlation between the two responses.

Figure 2 shows the posterior mean estimates of the expectations of the marginal distributions of the variables window and age at menarche as a function of a woman's year of birth.

[Figure 2 here]

The graph on the right-hand side of Figure 2 shows a decreasing effect of the year of birth on the expectation of age at menarche, suggesting that earlier menarche is associated with younger women. With respect to the effect on the expected value of the window, the graph on the left-hand side of Figure 2 shows a clear upward trend for women born before 1952, followed by a sharp drop. This is not surprising since this drop corresponds to younger women who had already reported going through menopause. Thus, these women are the ones who reached menopause relatively early, representing lower values for the window. The effects of the year of birth on the standard deviation of the marginal distributions of the window and menarche are shown in Figure 2.

[Figure 3 here]

A clear decreasing effect for both variables, window (left-hand side) and age at menarche (right-hand side), is observed in Figure 3. This decreasing effect in the standard deviation is to be expected since age at menarche and menopause are dates more easily remembered by younger women.

Finally, Figure 4 shows the estimated correlation between age at menarche and window as a function of the year of birth. A clear negative correlation is observed throughout the cohorts. This points to earlier ages of menarche as being associated with longer reproductive lifespan cycles. The stability of the negative correlation observed for the middle cohort, women born between 1931 and 1955 (the majority of women in our data), upholds the earlier suggested idea that not only is the decrease in a woman's age at menarche observed, but it also is marked by an increase in a woman's reproductive lifespan cycle.

[Figure 4 here]

As described in the previous section, the spatial effect was evaluated at each cohort, introducing varying coefficients in the predictor equation. The spatial effect was estimated at each cohort level separately: Level 1 - women born between [1920, 1930], Level 2 - women born between ]1930, 1955], and Level 3 - women born after 1955. Figures 5 through 8 show the spatial effects on the expectation and on the standard deviation of the window and age of menarche, respectively.

[Figures 5, 6, 7, 8 here]

From these figures we see very similar spatial effects on the variables window and age of menarche. The effect on the expected value of the window (Figure 5) and of the age at menarche (Figure 7) showed no significant spatial effects for the former and very few regions with significant spatial effects in the latter, when looking at the older cohort of women (age of birth before 1931, left graphs in Figures 5 and 7). For the middle cohort and especially in the younger women (Figures 5 and 7, middle and right graphs) the significant regions (both strictly positive and negative 95\% CI) are present throughout the entire central region of Portugal.

When looking at the spatial effect on the standard deviation of the variables window (Figure 6) and age at 
menarche (Figure 8), the similarities of these effects are even more evident. No significant effects in the older cohort (left graphs in Figures 6 and 8) and the interior of central Portugal shows a decreasing effect on the standard deviation of both variables, while the opposite effect was observed in the coastal regions. Regarding the correlation between age of menarche and a woman's reproductive lifespan cycle, Figure 9 shows almost no significant effects in all regions over the 3 cohorts of women.

[Figure 9 here]

\section{Discussion}

Since the coastal regions of central Portugal are in general more economically developed than its interior counterparts, the expectation was an increasing effect on the window and a decreasing effect on age of menarche in the east-west direction, especially for the younger women's cohorts.

The results showed a very clear downward trend for age at menarche together with an increase of a woman's reproductive lifespan cycle (Figure 2). More than analyzing the effect of the year of birth on the marginal behavior of age at menarche and a woman's reproductive lifespan cycle, the distributional multivariate model employed in this study allowed us to explore this effect on the correlation between these two variables. The negative effect achieved on the correlation, bringing into consonance the results observed for the age at menarche and a woman's reproductive lifespan cycle separately, proves the advantage of these models when modeling the effect of the covariates in the association between a bivariate response. As a final remark, this study substantiated once again the decline of the age of menarche over time, as was observed in the univariate study presented by (Duarte et al., 2014). In addition, for the first time, the upward trend of a woman's reproductive lifespan reported in the literature (Talma et al., 2013; Berman et al., 2012; Rigon et al., 2010; Cabanes et al., 2009; Nichols, 2006; Padez and Rocha, 2003) was detected.

Nevertheless, the expected east-west direction of the spatial effects was not corroborated by the results presented in the previous section. In particular, older women (born before 1931) showed a non-significant spatial effect on the expectation and on the standard deviation of the window. In addition, the results did not capture the expected spatial effect of earlier age of menarche and wider reproductive lifespan cycles associated with the coastal regions (Rodrigues, 1993; Duarte et al., 2014), not even for the younger women cohort. These results may be explained by external factors that were not taken into consideration in the model, which is an indication that spatial and social indicators are not the only factors associated with a woman's individual characteristics, namely age at menarche and the reproductive lifespan cycle of a woman.

A first possible external factor is migration movements within this central region of Portugal. For economic reasons, these movements in Portugal are more frequent in the east-west direction (Rees, P. et al., 1998). Thus, the chance that a woman who lives in the coastal area of the country is a non-native of that region is higher than for a woman who resides in the interior part of the country. Therefore, the current residence reported by women in our data set may not be a meaningful factor since it does not represent the true effect of the region where a woman spent her childhood and adolescence.

Secondly, the attendance and detection rates are in general affected by the existence of a parallel offer of breast cancer diagnostics, used frequently by woman with higher purchasing power. This is a distinguishing characteristic between the coastal and the interior regions, with a natural consequence of this self-selection factor, where the interior has a greater representativeness in the screening program.

Thirdly, the exposure to exogenous hormones like estrogen and progestogen, from which oral contraceptives are made, is an important factor that should be included in future analysis, since they may affect a woman's reproductive lifespan cycle.

Finally, as mentioned in Section 2, only the post-menopausal women who attended the Breast Cancer 
Screening Program in central Portugal were considered in this analysis, and therefore they mostly represent older women and those that have been part of the program for a longer time. Thus, younger women who have not reached menopause and have left the screening program before reaching menopause were excluded from this study. A challenge and an important contribution when dealing with this type of data is extending the distributional models to censored responses. This feature will be addressed in future research and will enable us to conduct further analyses with the inclusion of the pre-menopausal women in order to assess how the omission of the pre-menopausal women constitutes a significant bias in these first analysis.

Although the inclusion of the spatial effects was not sufficient to capture the complexity associated to the environmental factors, the results reflect the population where the data is selected and raised important questions related to what should be considered as environmental factor, whether a woman's place of residence or a woman's place of birth and her adolescent years. The latter information is not available in the breast cancer screening data provided by the Central Regional Nucleus of the Portuguese Cancer League for this study. This group is currently planning a serious of smaller studies addressing the impact of a screening program in women, where the inclusion of a woman's place of birth and her adolescent years may allow us to evaluate the true spatial effect in these two important variables, age at menarche and a woman's reproductive lifespan cycle, in studying breast cancer risk factors. 

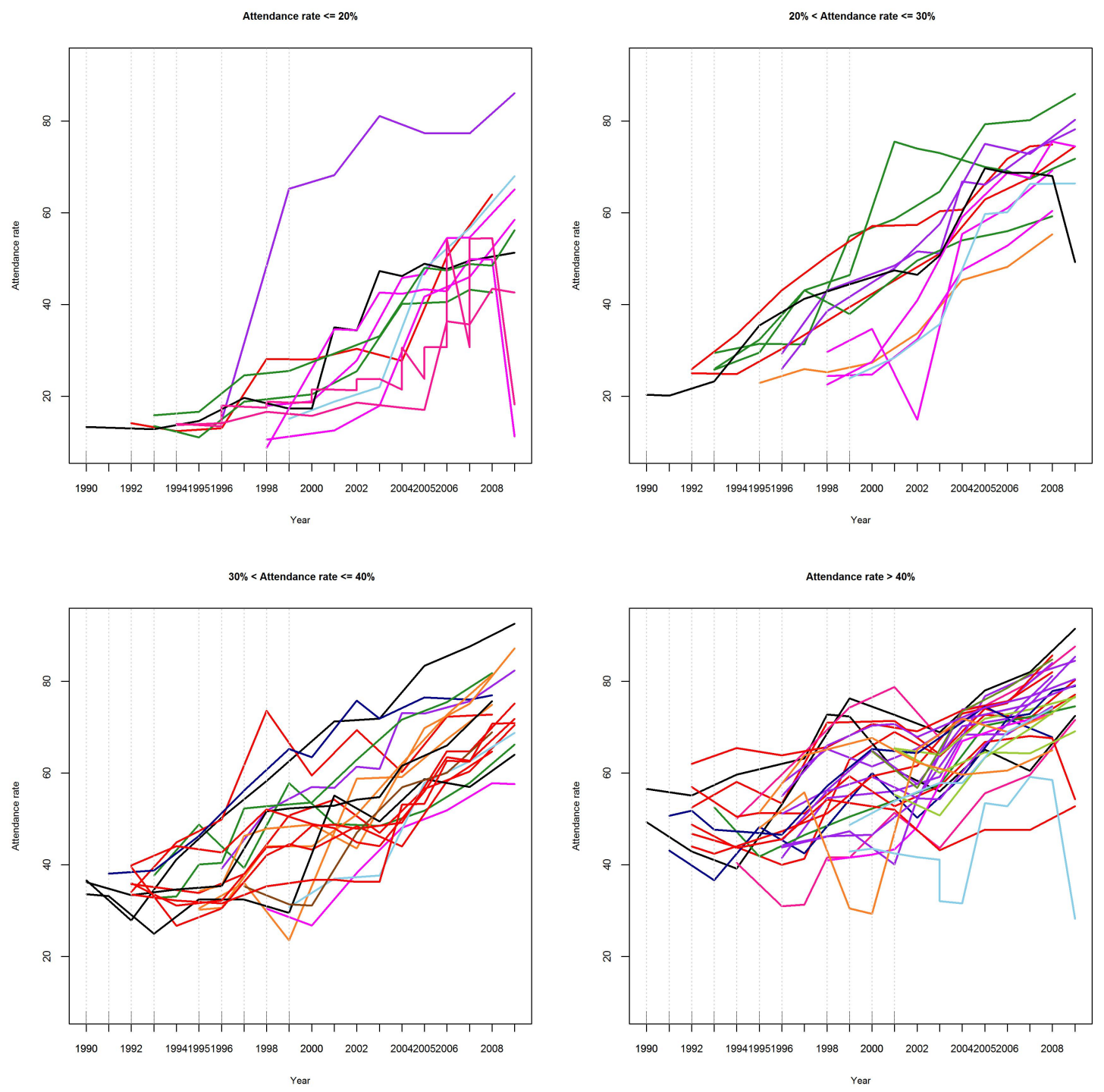

Figure 1 Evolution of the attendance rates for all BCSP-CB municipalities since they have entered the screening program, separated by their initial attendance rate. 

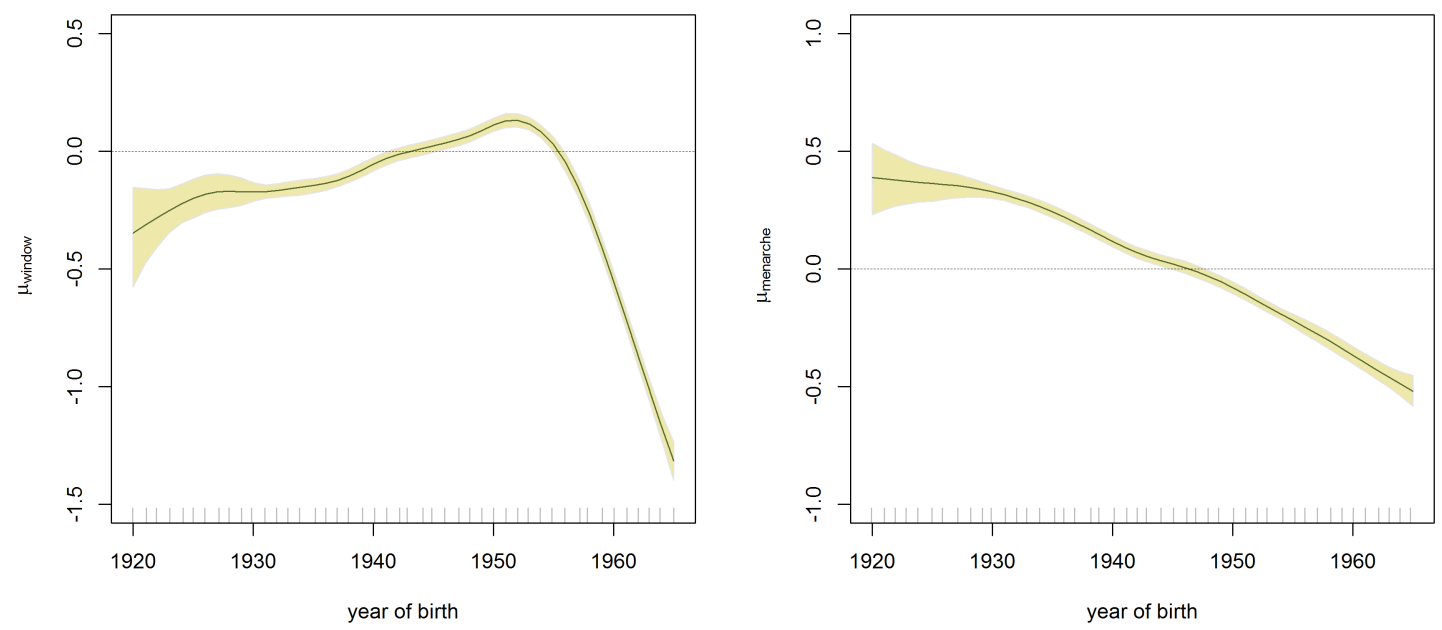

Figure 2 Year of birth effect - Posterior mean estimates of the expectation: $\mu_{\text {window }}$ (left) and on $\mu_{\text {menarche }}$ (right) with $95 \%$ pointwise credible intervals.
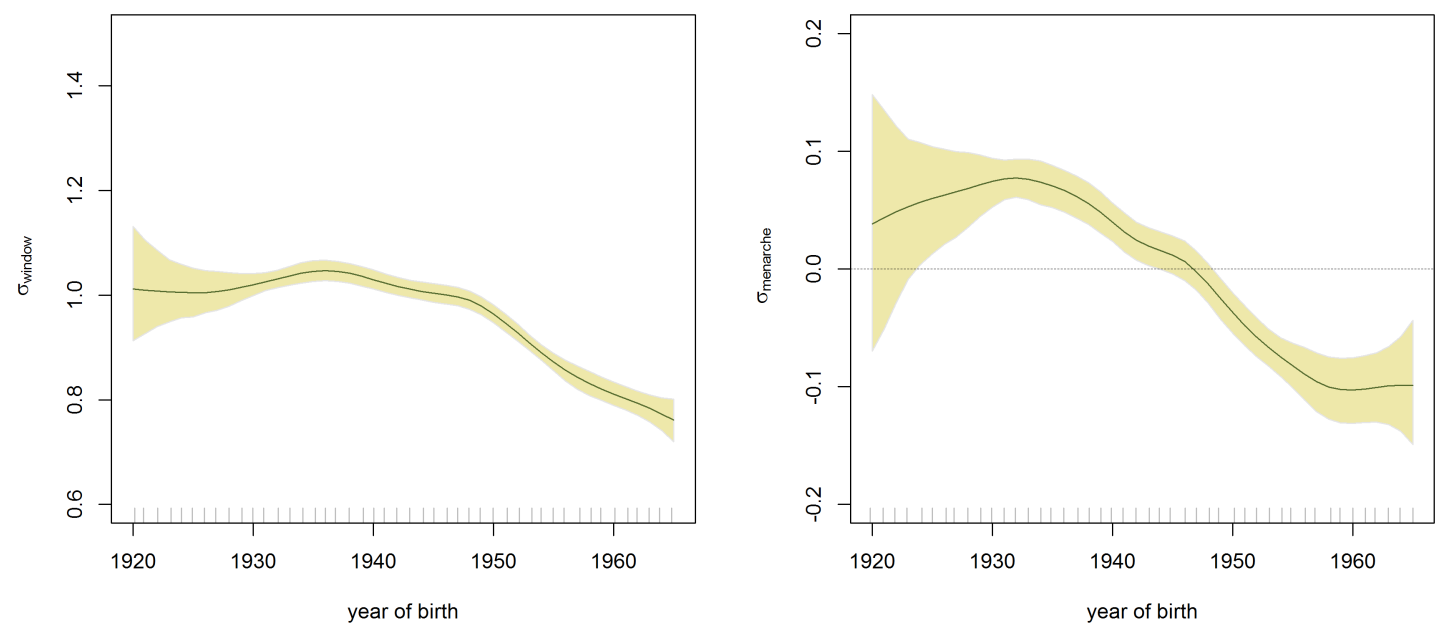

Figure 3 Year of birth effect - Posterior mean estimates of the standard deviation parameter: $\sigma_{\text {window }}$ (left) and on $\sigma_{\text {menarche }}$ (right) with $95 \%$ pointwise credible intervals. 


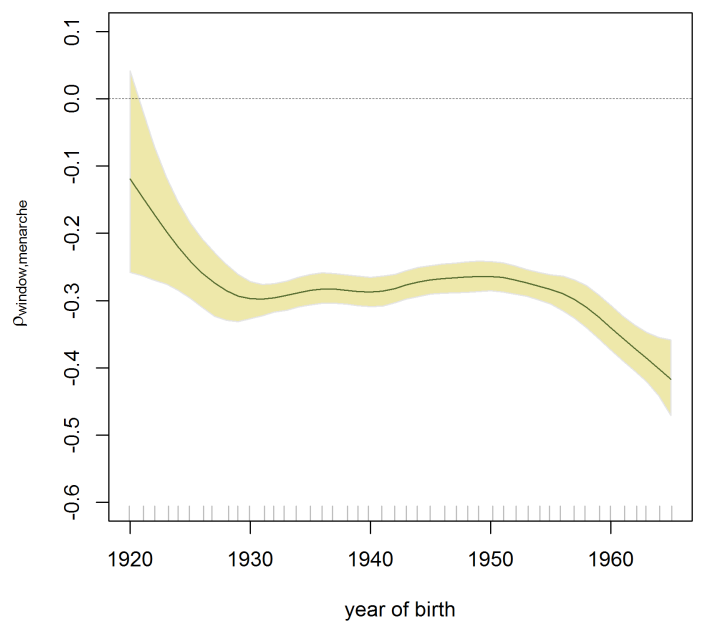

Figure 4 Year of birth effect - Posterior mean estimates of the correlation parameter $\rho_{\text {window,menarche }}$ with $95 \%$ pointwise credible intervals. 

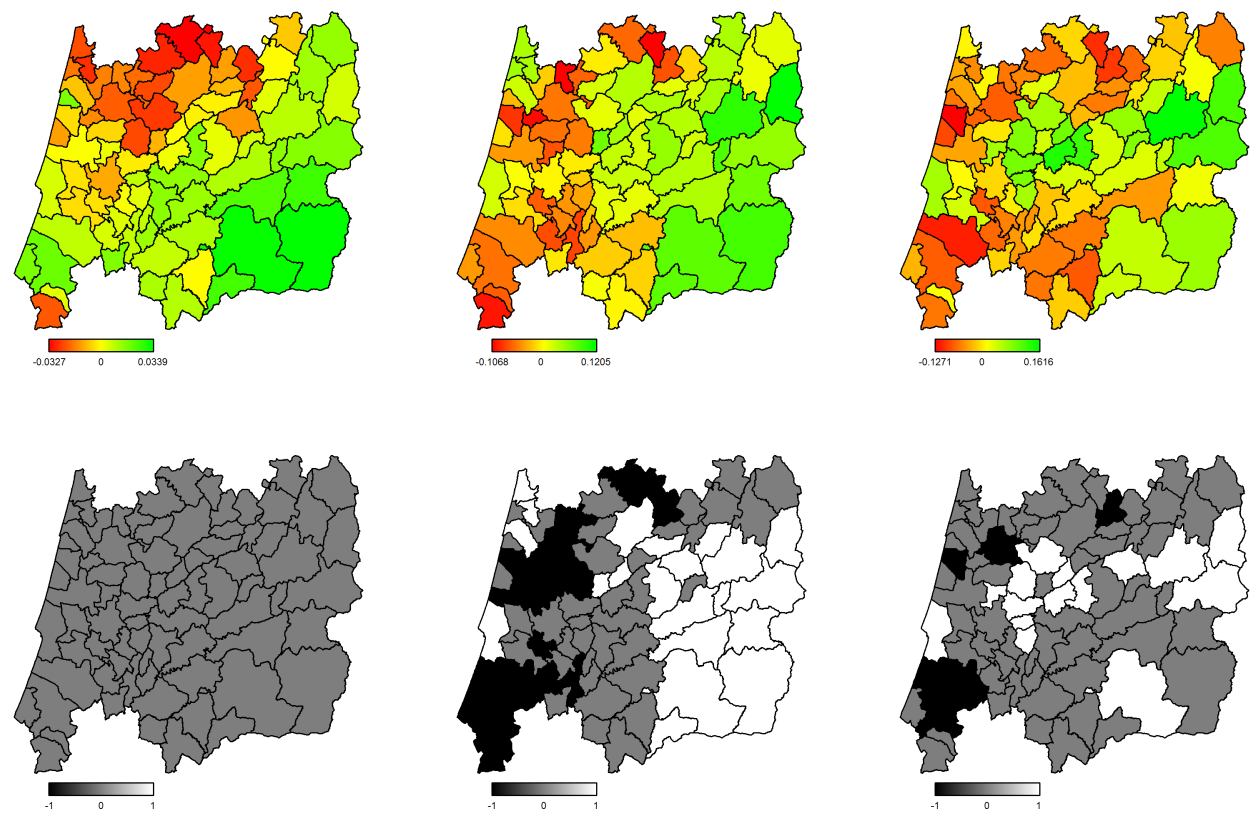

Figure 5 Spatial effect - Posterior mean estimates of the structured spatial effect on $\mu_{\text {window }}$ : Left: Level 1 - women born between [1920, 1930]; middle: Level 2 - women born between ]1930, 1955 ]; right: Level 3 - women born after 1955. The $95 \%$ related posterior probabilities are plotted below, where a value of 1 (white regions) corresponds to a strictly positive $95 \%$ credible interval, a value of -1 (black regions) to a strictly negative credible interval, and a value of 0 (gray regions) indicating that the corresponding credible interval contains 0 . 

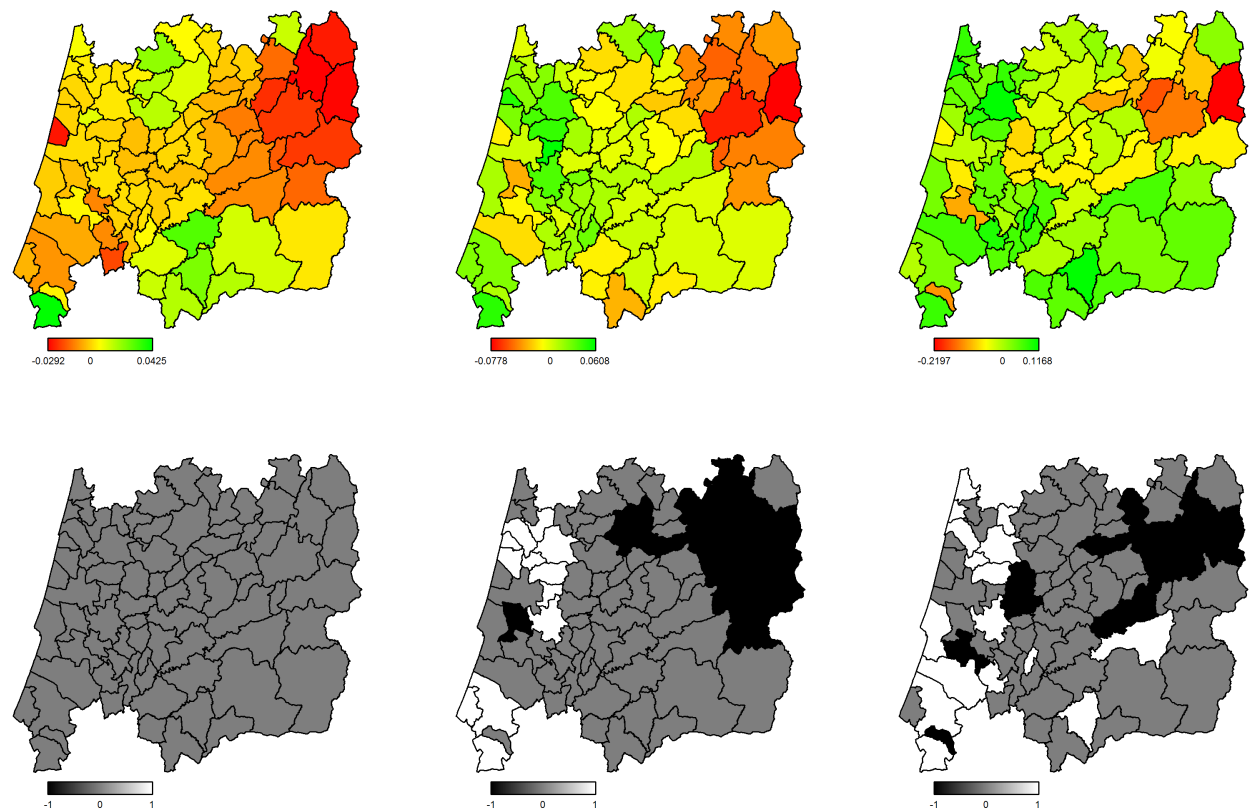

Figure 6 Spatial effect - Posterior mean estimates of the structured spatial effect on $\sigma_{\text {window }}$ : Left: Level 1 - women born between [1920, 1930]; middle: Level 2 - women born between ]1930, 1955 ]; right: Level 3 - women born after 1955. The $95 \%$ related posterior probabilities are plotted below, where a value of 1 (white regions) corresponds to a strictly positive $95 \%$ credible interval, a value of -1 (black regions) to a strictly negative credible interval, and a value of 0 (gray regions) indicating that the corresponding credible interval contains 0 . 

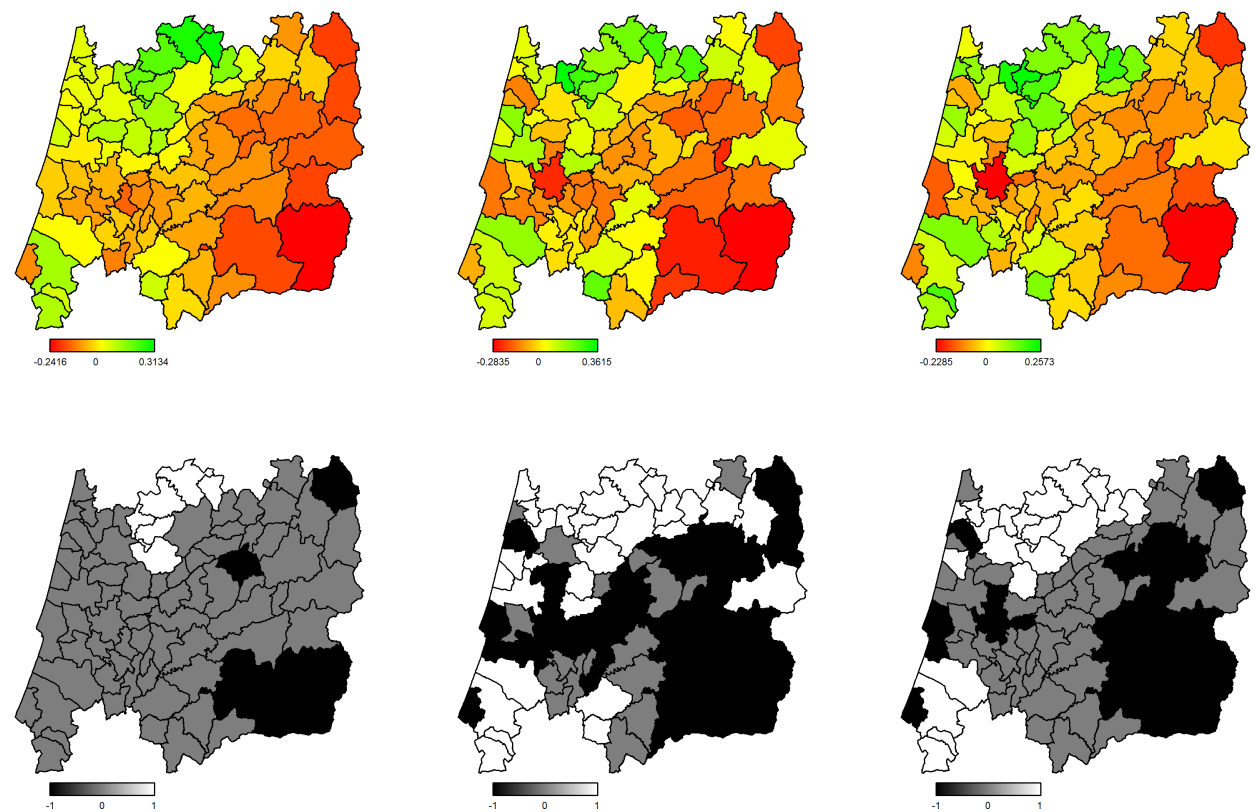

Figure 7 Spatial effect - Posterior mean estimates of the structured spatial effect on $\mu_{\text {menarche }}$ : Left: Level 1 - women born between [1920, 1930]; middle: Level 2 - women born between ]1930, 1955 ]; right: Level 3 - women born after 1955 . The $95 \%$ related posterior probabilities are plotted below, where a value of 1 (white regions) corresponds to a strictly positive 95\% credible interval, a value of -1 (black regions) to a strictly negative credible interval, and a value of 0 (gray regions) indicating that the corresponding credible interval contains 0 . 

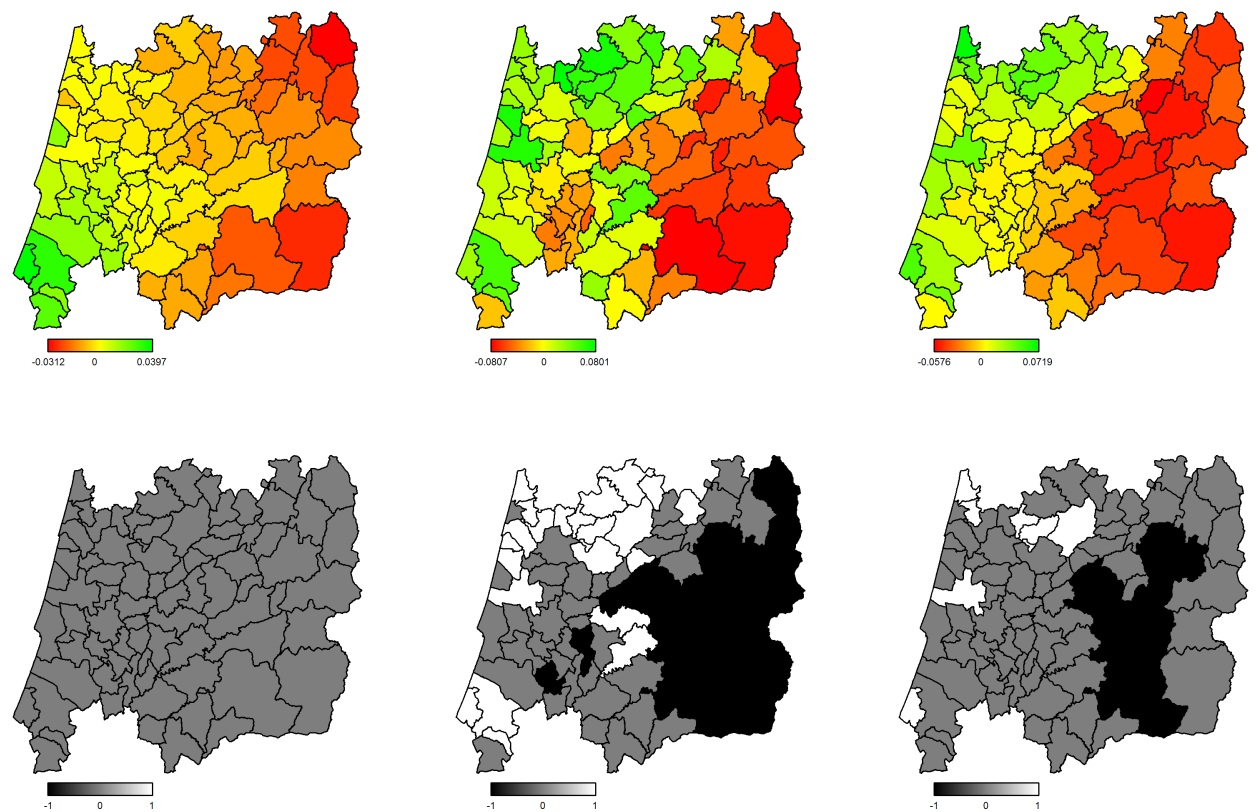

Figure 8 Spatial effect - Posterior mean estimates of the structured spatial effect on $\sigma_{\text {menarche }}$ : Left: Level 1 - women born between [1920, 1930]; middle: Level 2 - women born between ]1930, 1955 ]; right: Level 3 - women born after 1955 . The $95 \%$ related posterior probabilities are plotted below, where a value of 1 (white regions) corresponds to a strictly positive $95 \%$ credible interval, a value of -1 (black regions) to a strictly negative credible interval, and a value of 0 (gray regions) indicating that the corresponding credible interval contains 0 . 

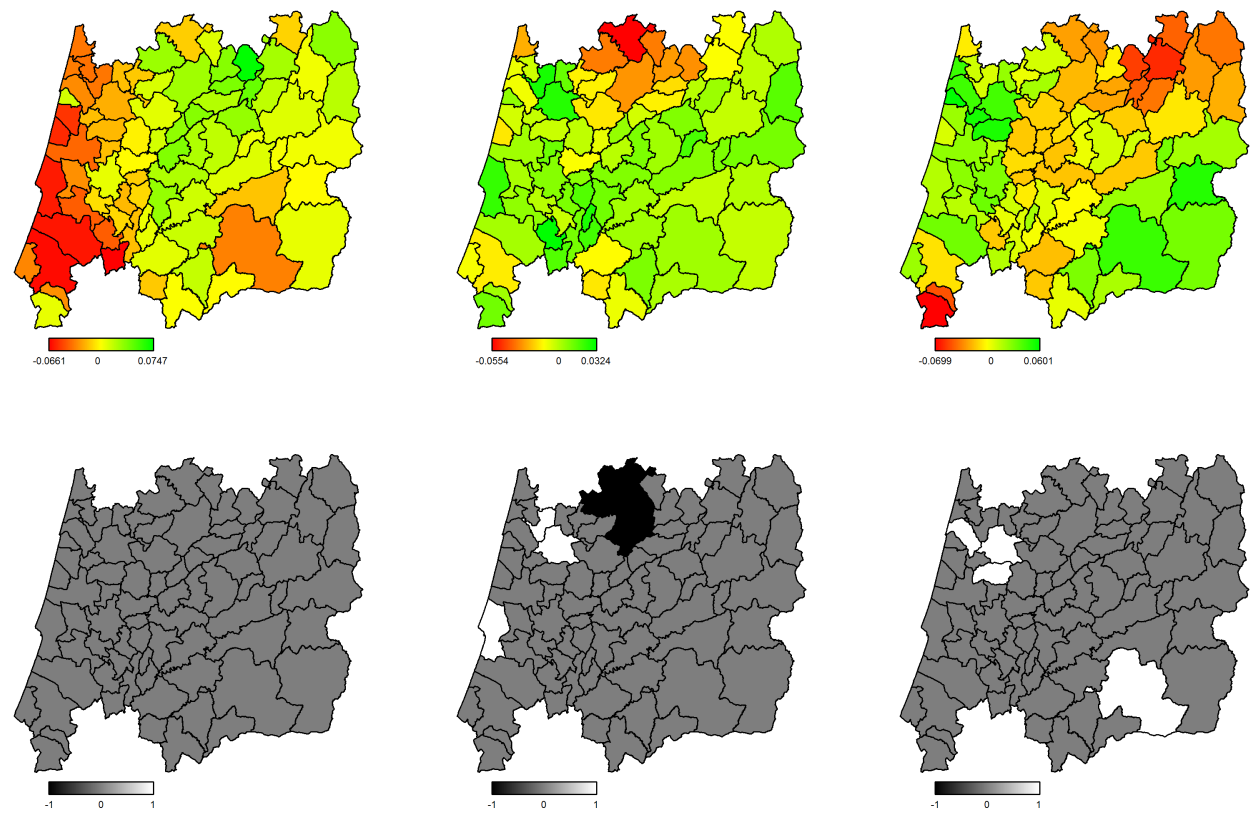

Figure 9 Spatial effect - Posterior mean estimates of the structured spatial effect on $\rho_{\text {window, menarche }}$ : Left: Level 1 - women born between [1920, 1930]; middle: Level 2 - women born between ]1930, 1955 ]; right: Level 3 - women born after 1955. The $95 \%$ related posterior probabilities are plotted below, where a value of 1 (white regions) corresponds to a strictly positive $95 \%$ credible interval, a value of -1 (black regions) to a strictly negative credible interval, and a value of 0 (gray regions) indicating that the corresponding credible interval contains 0 .

Table 1 Statistics of the variables in the study.

\begin{tabular}{lccc}
\hline Variable & Mean & Standard Deviation (SD) & Min-Max \\
\hline Birth year & 1946 & 9.8 & $1920-1965$ \\
Age of menarche & 13.3 & 8.0 & $8-18$ \\
Reproductive lifespan & 34.9 & 5.5 & $3-50$ \\
\hline
\end{tabular}

Table 2 Distribution of the women by cohorts

\begin{tabular}{cccccccc}
\hline \multirow{2}{*}{ Cohort } & \multirow{2}{*}{ Proportion (\%) } & \multicolumn{2}{c}{ Menarche age } & \multicolumn{2}{c}{ Menopause age } & \multicolumn{2}{c}{ Reproductive life span } \\
& & Mean & Median & Mean & Median & Mean & Median \\
\hline$[1920,1930]$ & 2.3 & 13.9 & 14 & 47.8 & 49 & 33.9 & 35 \\
]1930,1955] & 86.1 & 13.4 & 13 & 48.5 & 50 & 35.1 & 36 \\
$>1955$ & 11.6 & 12.8 & 13 & 46.0 & 47 & 33.2 & 34 \\
\hline
\end{tabular}

Acknowledgements This work was financed by POPH-QREN, the European Social Fund and national funds MCTES - Portuguese Ministry of Science, Technology and Higher Education through the research project SFRH/BD/64761/2009, the Spanish Ministry of Science and Innovation grant MTM2015-69068-REDT, and the 2014 
Competitive Call for "Acciones Conjuntas Hispano-Alemans/PPP Spain" in cooperation with the Foundation for the International Promotion of Spanish Universities Unversidad.es. This study also was supported by the projects MTM2014-52975-C2-1-R co-financed by the Ministry of Economy and Competitiveness (SPAIN) and the European Regional Development Fund (FEDER) and German Research Foundation (DFG) grant KN 922/4-2.

\section{Conflict of Interest The authors have declared no conflict of interest.}

\section{References}

Belitz, C., Brezger, A., Klein, N., Kneib, T., Lang, S., Umlauf, N.(2015) BayesX - Software for Bayesian inference in structured additive regression models. Version 3.0.2. Available from http: / / www.bayesx.org

Berman, T., Levine, H.,Gamzu, R., Grotto, I. (2012). Trends in reproductive health in Israel:implications for environmental health policy. Israel Journal of Health Policy Research 1, 34.

Brezger, A. and Lang, S. (2006). Generalized additive regression based on Bayesian P-splines. Computational Statistics \& Data Analysis 50, 967-991.

Cabanes, A., Ascunce ,N., Vidal, E., Ederra, M., Barcos, A., Erdozain, N., Lope, V., Pollán, M.(2009). Decline in age at menarche among Spanish women born from 1925 to 1962. BMC Public Health 9, 449.

Collaborative Group on Hormonal Factors in Breast Cancer (2012). Menarche, menopause, and breast cancer risk: individual participant meta-analysis,including 118964 women with breast cancer from 117 epidemiological studies. Lancet Oncol 2012 13(11),1141-1151; published online Oct 17. url: http://dx.doi.org/10.1016/S1470-2045 (12)70425-4.

Duarte E., de Sousa B., Cadarso-Suárez C., Rodrigues V., Kneib T.(2014) Structured additive regression (STAR) modeling of age of menarche and the age of menopause in breast cancer screening program. Biometrical Journal, 56, 416-427

Eilers, P. H. C. and Marx, B. D. (1996). Flexible smoothing using B-splines and penalties (with comments and rejoinder). Statiscal Science 11, 89-121.

Fahrmeir, L., Kneib, T. and Lang, S. (2004). Penalized structured additive regression for space-time data: a Bayesian perspective. Statistica Sinica 14, 731-761.

Fahrmeir, L. and Lang, S. (2001). Bayesian Inference for Generalized Additive Mixed Models Based on Markov Random Fields Priors. Applied Statistics 50(2), 201-220.

Hsieh, C.C., Trichopoulos, D., Katsouyanni, K., Yuasa, S.(1990). Age at menarche, age at menopause, height and obesity as risk factors for breast cancer: associations and interactions in an international case-control study. International Journal of Cancer 46(5), 796-800.

Kelsey, J. L., Horn-Ross, P. L. (1993). Breast cancer: magnitude of the problem and descriptive epidemiology. Epidemiologic Reviews 15(1), 7-16.

Kelsey, J. L., Gammon, M.D., John, E.M. (1993). Reproductive factors and breast cancer Epidemiologic Reviews 15, 36-47.

Klein N., Kneib T., Lang S., Sohn A. (2015). Bayesian structured additive distributional regression with an application to regional income inequality in Germany. Annals of Applied Statistics 20159 (2), 569591. doi: $10.1111 /$ rssc. 12090

Klein N., Kneib T., Klasen S. and Lang S. (2015). Bayesian structured additive distributional regression for multivariate responses. Journal of the Royal Statistical Society: Series C (Applied Statistics), 64, 1024-1052. doi:10.1214/15-AOAS823

Lambe, M.(2010). Reproductive Factors. In: Breast Cancer Epidemiology (eds. Christopher I. Li), Springer Science+Business Media, New York, 119-135.

Lang, S., Brezger, A. (2004). Bayesian P-splines. Journal of Computational and Graphical Statistics 13, $183-212$.

McPherson, K., Steel, C. M., Dixon, J. M.(2000). Breast cancer - epidemiology, risk factors, and genetics. British Medical Journal 321, 624-628.

Nichols, H. B., Trentham-Dietz, A., Hampton, J. M., Titus-Ernstoff, L., Egan, K. M., Willett, W. C., Newcomb, P. A.(2006). From Menarche to Menopause: Trends among US Women Born from 1912 to 1969. American Journal of Epidemiology 164(10), 1003-1011. doi: 10.1093/aje/kwj282 
Padez, C., Rocha, M. A. (2003). Age at menarche in Coimbra (Portugal) school girls: a note on the secular changes. Annals of Human Biology 30(5), 622-632.

Rees, P., Carrilho, M.J., Joo, P., Durham, H., Kupiszewski, M. (1998). Internal Migration and Regional Population Dynamics in Europe: Portugal Case Study. Working Paper. School of Geography, University of Leeds. Available from url:http: / /www.geog.leeds.ac.uk/wpapers/

Rigon, F., Bianchin, L., Bernasconi, S., Bona, G., Bozzola, M., Buzi, F.,et al. (2010). Update on Age at Menarche in Italy: Toward the Leveling Off of the Secular Trend. Journal of Adolescent Health 46, 238-244.

Rodrigues, V. (1993). Geographical epidemiology of cancer. Application of empirical Bayesian estimation to the analysis of the geographical distribution of mortality from malignant tumors in Portugal. $\mathrm{PhD}$ thesis, Faculty of Medicine, University of Coimbra, Portugal.

Rue, H. and Held, L. (2005). Gaussian Markov Random Fields. Chapman \& Hall/CRC, Boca Raton, FL.

Talma, H., Schönbeck, Y., van Dommelen, P., Bakker, B., van Buuren, S., HiraSing, R. A. (2013). Trends in Menarcheal Age between 1955 and 2009 in the Netherlands. PLoS ONE 8(4): e60056. doi:10.1371/journal.pone.0060056.

\section{Supporting information}

The supplementary material for this article includes the dataset (datafile.txt), the graph and boundary files (PTCentralN.gra, PTCentralN.bnd). The BayesX code used to perform the models described in Section 3, are available in the scriptBayesX.txt and the R commands to generate the reults plots, are available in the $\mathrm{R}$ file plotresults.R. 
This article is protected by copyright. All rights reserved. 


\section{University Library}

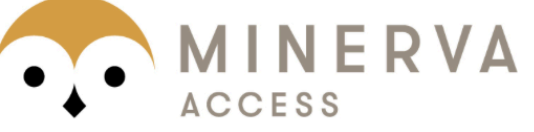

A gateway to Melbourne's research publications

Minerva Access is the Institutional Repository of The University of Melbourne

Author/s:

Duarte, E;de Sousa, B;Cadarso-Suarez, C;Klein, N;Kneib, T;Rodrigues, V

Title:

Studying the relationship between a woman's reproductive lifespan and age at menarche using a Bayesian multivariate structured additive distributional regression model

Date:

2017-11-01

Citation:

Duarte, E., de Sousa, B., Cadarso-Suarez, C., Klein, N., Kneib, T. \& Rodrigues, V. (2017). Studying the relationship between a woman's reproductive lifespan and age at menarche using a Bayesian multivariate structured additive distributional regression model. BIOMETRICAL JOURNAL, 59 (6), pp.1232-1246. https://doi.org/10.1002/bimj.201600245.

Persistent Link:

http://hdl.handle.net/11343/293119 